PROCEEDINGS OF THE

AMERICAN MATHEMATICAL SOCIETY

Volume 125, Number 5, May 1997, Pages 1377-1379

S 0002-9939(97)03767-2

\title{
PEAK SET WITHOUT PEAK POINTS
}

\author{
KRZYSZTOF JAROSZ
}

(Communicated by Theodore W. Gamelin)

\begin{abstract}
We give an example of a natural Banach function algebra on the unit disc such that a smaller disc is a peak set for the algebra, but it does not contain any peak point.
\end{abstract}

A Banach function algebra on a compact Hausdorff space $X$ is a Banach algebra $A$ consisting of continuous functions on $X$, such that $A$ separates points of $X$ and contains the constant functions. If the norm of the algebra $A$ coincides with the sup norm on $X$, it is called a uniform algebra. If any linear and multiplicative functional on $A$ is of the form $f \mapsto f(x)$ for some $x \in X$, the algebra is called natural. A subset $K$ of $X$ is a peak set for $A$ if there is an $f \in A$ such that $f \equiv 1$ on $K$ and $|f(x)|<1$ for $x \notin K$; if $K=\left\{x_{0}\right\}$, then $x_{0}$ is a peak point. If no proper subset of $K$ is a peak set we call it a minimal peak set for $A$. It is well known [2] that if $A$ is a uniform algebra on a metrizable set $X$ then any peak set contains a peak point. In [1] H. G. Dales constructed a natural Banach function algebra on a compact subset of $\mathbb{C}^{2}$ having a peak set without any peak point. T. G. Honary [3] provided an example in $\mathbb{R}^{3}$, however his algebra is not natural. In this note we give a very simple example of a natural Banach function algebra on the unit disc with peak sets not containing any peak point.

Let $K$ be an open nonempty subset of the complex plane $\mathbb{C}$. By $C^{1}(K)$ we denote the algebra of all bounded complex valued functions on $\bar{K}$ with continuous and bounded first order partial derivatives on $K . C^{1}(K)$ is a Banach function algebra on $\bar{K}$ if equipped with the norm

$$
\|f\|=\|f\|_{\infty}+\left\|f_{x}\right\|_{\infty}+\left\|f_{y}\right\|_{\infty},
$$

where $\|\cdot\|_{\infty}$ is the sup norm. By $A(K)$ we denote the uniform algebra of all continuous functions on $\bar{K}$ which are analytic on $K$. We put $\mathbb{D}_{r}=\{z \in \mathbb{C}:|z|<r\}$ and $\mathbb{C}_{-}=\{z: \operatorname{Re} z<0\}$.

Theorem 1. Let $A$ be a subalgebra of $C^{1}\left(\mathbb{D}_{1}\right)$ consisting of all functions which are analytic on $\mathbb{D}_{\frac{1}{2}}$. Then $\overline{\mathbb{D}_{\frac{1}{2}}}$ is a minimal peak set for $A$; in particular $\overline{\mathbb{D}_{\frac{1}{2}}}$ does not contain any peak point.

Lemma 2. There is no $h \in A\left(\mathbb{D}_{1}\right)$ such that $h(1)=0$ and $|1+(z-1) h(z)|<1$ for $z \neq 1$.

Proof of the lemma. Assume that such a function does exist. If we compose $1+$ $(z-1) h(z)$ with a suitable fractional linear transformation we get an $f \in A\left(\mathbb{C}_{-}\right)$

Received by the editors November 8, 1995.

1991 Mathematics Subject Classification. Primary 46J10.

(C)1997 American Mathematical Society 
such that $f(z)=1+z g(z), g(0)=0$, and $|f(z)|<1$ for $z \in \overline{\mathbb{C}_{-}} \backslash\{0\}$. Let $\alpha$ be a positively oriented curve consisting of the segment from $-i$ to $+i$ and the half of the unit circle contained in $\mathbb{C}_{-}$. We shall show that $\gamma=g \circ \alpha$ has negative orientation, which will contradict the fact that $g$ is analytic.

For any $z \in \overline{\mathbb{C}_{-}} \backslash\{0\}$ we have $\operatorname{Re} z g(z)<0$, so

- as $z$ moves up from 0 to $+i$ along the curve $\alpha, \operatorname{Im} g(z)>0$,

- as $z$ moves along the half circle of $\alpha, g(z)$ is in $\mathbb{C} \backslash\{z: \operatorname{Re} z \leq 0, \operatorname{Im} z=0\}$,

- as $z$ moves from $-i$ to 0 on the vertical line, $\operatorname{Im} g(z)<0$.

Consequently, if $z_{0}$ is a positive real number such that the segment $\left(0, z_{0}\right]$ does not intersect $\gamma$, then the orientation of $\gamma$ around $z_{0}$, as well as around any point from $\left(0, z_{0}\right]$, is negative.

Proof of the theorem. It is well known that there is a $C^{1}$-function, and even a $C^{\infty}$ function on $\mathbb{D}_{1}$, peaking exactly on $\overline{\mathbb{D}_{\frac{1}{2}}}$; thus $\overline{\mathbb{D}_{\frac{1}{2}}}$ is a peak set.

Assume $\frac{1}{2}$ is a peak point for $A$ and let $f \in A$ be a function peaking at $\frac{1}{2}$. Put $u=\operatorname{Re} f, v=\operatorname{Im} f$. Since $u^{2}+v^{2}$ has the maximum at $\frac{1}{2}$, and $u\left(\frac{1}{2}\right)=1, v\left(\frac{1}{2}\right)=0$, it follows that $u_{x}\left(\frac{1}{2}\right)=u_{y}\left(\frac{1}{2}\right)=0$. Since the partial derivatives are continuous, and $f$ satisfies the Cauchy-Riemann equations on $\mathbb{D}_{\frac{1}{2}}$, we get $v_{x}\left(\frac{1}{2}\right)=v_{y}\left(\frac{1}{2}\right)=0$. Hence

$$
g(z) \stackrel{d f}{=} \frac{f(z)-1}{z-\frac{1}{2}} \rightarrow 0, \quad \text { as } z \rightarrow \frac{1}{2}
$$

so $g \in A\left(\mathbb{D}_{\frac{1}{2}}\right), g\left(\frac{1}{2}\right)=0$, and $f(z)=1+\left(z-\frac{1}{2}\right) g(z)$ has a strict maximum at $\frac{1}{2}$. This contradicts the lemma. Hence $\overline{\mathbb{D}_{\frac{1}{2}}}$ does not contain any peak point.

What remains to show is that $\overline{\mathbb{D}_{\frac{1}{2}}}$ does not contain any proper peak set. Assume $K \varsubsetneqq \overline{\mathbb{D}_{\frac{1}{2}}}$ is a peak set and let $F \in A$ be a corresponding function peaking on $K$. Notice that $K$ cannot contain the entire circle $\partial \mathbb{D}_{\frac{1}{2}}$. Let $z_{0} \in K \cap \partial \mathbb{D}_{\frac{1}{2}}$ and $z_{1} \in \partial \mathbb{D}_{\frac{1}{2}} \backslash K$. For any $w \in \partial \mathbb{D}_{\frac{1}{2}} \backslash\left\{z_{0}\right\}$ let $\varphi_{w}$ be a $C^{1}$-automorphism of $\overline{\mathbb{D}}$ mapping $\overline{\mathbb{D}_{\frac{1}{2}}}$ onto itself, analytic on $\mathbb{D}_{\frac{1}{2}}$, and such that $\varphi_{w}\left(z_{0}\right)=z_{0}$ and $\varphi_{w}(w)=z_{1}$. Such an automorphism can be obtained by smoothly extending a suitable fractional linear automorphism of $\mathbb{D}_{\frac{1}{2}}$. Sets of the form $U_{w}=\left\{z \in \overline{\mathbb{D}_{\frac{1}{2}}} \backslash\left\{z_{0}\right\}:\left|F \circ \varphi_{w}(z)\right|<1\right\}$ form an open cover of $\partial \mathbb{D}_{\frac{1}{2}} \backslash\left\{z_{0}\right\}$. Let $\left\{w_{1}, w_{2}, \ldots\right\}$ be such that $\bigcup_{j=1}^{\infty} U_{w_{j}}=\partial \mathbb{D}_{\frac{1}{2}} \backslash\left\{z_{0}\right\}$. Put $G=\sum_{j=1}^{\infty} \frac{F \circ \varphi_{w_{j}}}{2^{j}\left\|F \circ \varphi_{w_{j}}\right\|}$. This function $G$ belongs to $A$ and peaks exactly at $z_{0}$, which contradicts the previous part of the proof.

Notice that essentially the same proof can be repeated to provide a slightly more general example:

Let $\Omega_{j}, j=1,2, \ldots$, be a sequence of open subsets of $\mathbb{D}_{1}$ such that for any $j, \overline{\Omega_{j}}$ has an open neighborhood contained in $\mathbb{D}_{1}$ not intersecting $\bigcup_{i \neq j} \Omega_{i}$, and such that $\partial \Omega_{j}$ is a union of finitely many smooth Jordan curves. Let $A$ be a subalgebra of $C^{1}\left(\mathbb{D}_{1}\right)$ consisting of all functions which are analytic on $\bigcup \Omega_{j}$. Then for any $j, \overline{\Omega_{j}}$ is a peak set not containing any peak point.

However, it is not clear whether the result can be further extended to Swiss cheese type domains to provide an example of a natural Banach function algebra where the union of all nontrivial minimal peak sets is dense in the unit disc. The 
difficulty, in general, is not in proving that $\Omega_{j}$ does not contain any peak point, but in proving that $\overline{\Omega_{j}}$ is a peak set.

\section{REFERENCES}

[1] H. G. Dales. Boundaries and peak points for Banach function algebras. Proc. London Math. Soc., 22(3):121-136, 1971. MR 43:2510

[2] T. W. Gamelin. Uniform Algebras. Chelsea Pub. Comp., New York, 1984. MR 53:14137

[3] T. G. Honary. An example of a Banach function algebra having a peak set without any peak point. In Proceedings of The Fifth Analysis Seminar at Shiraz University, pages 26-32, 1990.

Department of Mathematics, Bowling Green State University, Bowling Green, Ohio 43403

Current address: Department of Mathematics \& Statistics, Southern Illinois University, Edwardsville, Illinois 62026

E-mail address: kjarosz@siue.edu 